\title{
Drug treatments in Alzheimer's disease
}

\author{
Authors: Robert Briggs, ${ }^{A}$ Sean P Kennelly ${ }^{B}$ and Desmond O'Neill ${ }^{\mathrm{C}}$
}

Despite the significant public health issue that it poses, only five medical treatments have been approved for Alzheimer's disease (AD) and these act to control symptoms rather than alter the course of the disease. Studies of potential diseasemodifying therapy have generally been undertaken in patients with clinically detectable disease, yet evidence suggests that the pathological changes associated with $A D$ begin several years before this. It is possible that pharmacological therapy may be beneficial in this pre-clinical stage before the neurodegenerative process is established. Techniques providing earlier diagnosis, such as cerebrospinal fluid biomarkers and amyloid positron emission tomography neuroimaging, are key to testing this theory in clinical trials. Recent results from trials of agents such as aducanumab are encouraging but must also be interpreted with caution. Such medicines could potentially delay the onset of dementia and would therefore markedly reduce its prevalence. However, we currently remain a good distance away from clinically available disease-modifying therapy.

KEYWORDS:: Alzheimer's disease, pharmacological therapy, amyloid

\section{Introduction}

Dementia is a general term for a decline in cognitive ability severe enough to interfere with daily life. Alzheimer's disease (AD) accounts for almost three-quarters of cases of dementia, with the remainder accounted for by vascular dementia (VaD), mixed Alzheimer's and VaD, dementia with Lewy bodies, and frontotemporal dementia.

To many clinicians, the contrast between the significant advances in the last two decades in medical treatment for a wide range of illnesses, including targeted therapies, such as herceptin, for many cancers, as well as revolutionary combination drug therapies for HIV, and the lack of progress

Authors: Aresearch fellow, Centre for Ageing, Neuroscience and the Humanities, Trinity Centre for Health Sciences, Tallaght Hospital, Dublin, Ireland; ${ }^{B}$ consultant geriatrician and stroke physician, Centre for Ageing, Neuroscience and the Humanities, Trinity Centre for Health Sciences, Tallaght Hospital, Dublin, Ireland; ' ${ }^{\text {consultant }}$ geriatrician and professor of medical gerontology, Centre for Ageing, Neuroscience and the Humanities, Trinity Centre for Health Sciences, Tallaght Hospital, Dublin, Ireland in the pharmacological treatment of dementia due to $\mathrm{AD}$ and other causes is surprising. However, it is increasingly clear that whereas the former illnesses are almost invariably well-defined and circumscribed disease entities, the syndrome of dementia is a multifactorial condition for most patients, ${ }^{1}$ and even within diagnostic entities such as $\mathrm{AD}$, it is likely that there are subclassifications of therapeutic significance.

Both of these findings have significant ramifications for treatment. The enmeshing of vascular disease and neurodegenerative illness in later life mean that dementia in later life is best viewed as a geriatric syndrome. The relevance of this conceptualisation is that geriatric syndromes, such as falls, rarely respond to single interventions, and pharmacological interventions are likely to succeed best in terms of one component of a package, which might include medication review, nutrition and exercise intervention, and cognitive stimulation/training, an approach supported by a recent multi-modal intervention study among older people at risk of developing dementia. ${ }^{2}$

Despite the significant public health issue that dementia poses, to date only five medical treatments have been approved for $\mathrm{AD}$, involving only two classes of drugs, and these act to control symptoms rather than alter the course of the disease. Additionally, relatively few clinical trials have been undertaken in $\mathrm{AD}$ in the last decade, and these have had a $99.6 \%$ failure rate. $^{3}$ As a result, the goal of disease-modifying therapy remains elusive, with the currently available medications acting to control symptoms only. In this review, we summarise current pharmacological treatment for $\mathrm{AD}$, as well as highlighting potential future therapies, while reflecting on the underlying aetiological mechanisms on which these treatments are based.

\section{Neurodegenerative pathways implicated in AD}

Several overlapping mechanisms have been proposed to explain the underlying pathology of $\mathrm{AD}$, and both current and potential future treatments are based on modification of these pathways (Fig 1).

\section{Amyloid cascade hypothesis}

The amyloid hypothesis of AD began to gain traction in the 1990 s, and centres on abnormal processing of the amyloid precursor protein (APP), leading to production of amyloidbeta $(\mathrm{A} \beta) .{ }^{4}$ Secretase enzymes cleave APP and aberrancy of this process, specifically mutations in gamma and betasecretases, can lead to the abnormal production of $A \beta .{ }^{5} \mathrm{~A} \beta$ 
Fig 1. Aetiology of Alzheimer's disease with therapeutic targets. A - secretase enzyme inhibitors; B - NMDA receptor modulators, eg memantine; C-immunotherapy, including immunisation and direct antiamyloid therapy, including monoclonal antibodies; D - anti-tau therapy; $\mathrm{E}$ - anti-inflammatory treatments, including NSAIDs; $\mathrm{F}$ - anticholinesterase inhibitors, eg donepezil. APP = amyloid precursor protein; NFTs = neurofibrillary tangles; $\mathrm{NMDA}=\mathrm{N}$-methyl-D aspartate; NSAIDs = non-steroida anti-inflammatory drugs.

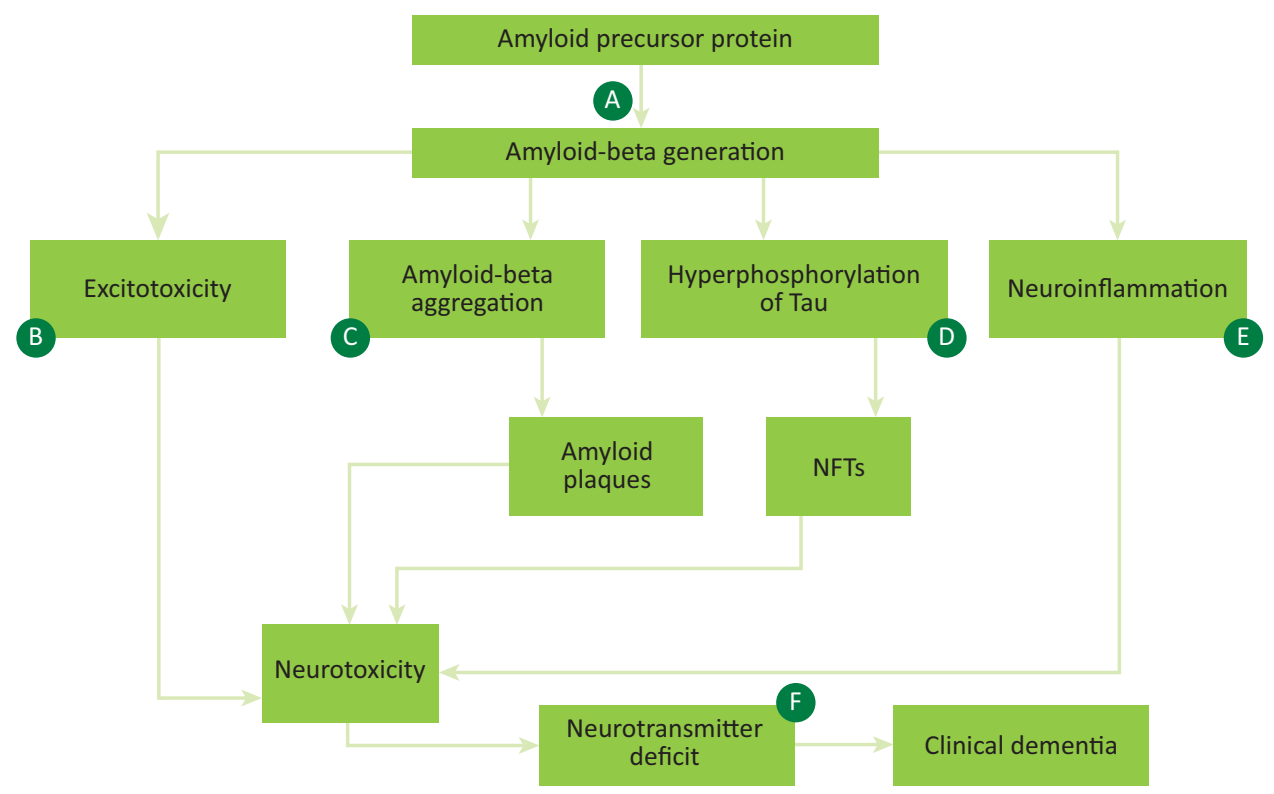

can then trigger a cascade leading to synaptic damage and neuron loss, and ultimately to the pathological hallmarks of AD: amyloid plaques and neurofibrillary tangles (NFTs) composed of hyperphosphorylated tau protein, with resulting neurodegeneration (Fig 1). ${ }^{6}$

\section{Tau hypothesis}

Tau is a protein expressed in neurons that normally functions in the stabilisation of microtubules in the cell cytoskeleton. ${ }^{7}$ Hyperphosphorylation causes it to accumulate into these NFT masses inside nerve cell bodies. These tangles then aberrantly interact with cellular proteins, preventing them from executing their normal functions. Hyperphosphorylation occurs downstream of $A \beta$, with research suggesting that accumulation of $A \beta$ may initiate this process. ${ }^{8}$ Additionally, there is evidence that toxic tau can enhance $A \beta$ production via a feedback loop mechanism. ${ }^{9}$

\section{Cholinergic hypothesis}

An initial breakthrough in AD came in the 1970s with the demonstration of a cholinergic deficit in the brains of patients with $\mathrm{AD}$, mediated by deficits in the enzyme choline acetyltransferase. ${ }^{10}$ This, along with the recognition of the role of acetylcholine in memory and learning, led to the cholinergic hypothesis of $\mathrm{AD}$ and stimulated attempts to therapeutically increase cholinergic activity. Cholinergic depletion is a late feature of the neurodegenerative cascade. Cholinesterase inhibitors block the cholinesterase enzyme, which breaks down acetyl choline at the synaptic cleft, potentiating cholinergic transmission.

\section{Excitotoxicity}

Excitotoxicity, defined as overexposure to the neurotransmitter glutamate, or overstimulation of its $\mathrm{N}$-methyl-D-aspartate
(NMDA) receptor, plays an important role in the progressive neuronal loss of AD. ${ }^{11}$ It is thought that loss of cholinergic neurons is affected by this process, resulting in excessive influx of calcium into cells.

\section{Other important aetiological mechanisms}

\section{Vascular disease}

While traditionally it was felt that vascular disease was the underlying factor in the development of $\mathrm{VaD}$, it is clear that vascular burden also plays a role in $\mathrm{AD}$ pathogenesis, and that there is significant overlap between these two dementia subtypes. ${ }^{12}$ Vascular risk factors, such as high BMI, smoking, hypercholesterolemia and hypertension, have been associated with an increased risk of developing clinical AD. ${ }^{13}$ While vascular lesions such as cerebral amyloid angiopathy and white matter hyperintensities are common in patients with $\mathrm{AD},{ }^{14}$ hypertension is also associated with the development of specific neuropathological hallmarks of AD such as NFTs, ${ }^{15}$ and this association appears to be stronger when hypertension is present in mid rather than late life. ${ }^{16}$ Thus, vascular disease may directly affect amyloid plaques or NFTs by increasing their formation or reducing their elimination from the brain. ${ }^{17}$

Given these findings, it would seem to follow that control of vascular risk factors may slow the rate of decline of cognition in patients with $\mathrm{AD}$; however, this is not yet supported by data from randomised controlled trials. ${ }^{18}$

\section{Diabetes and hyperinsulinaemia}

There is a well-established association between diabetes and $\mathrm{AD}^{19}$ and while vascular burden undoubtedly plays a role in this, it appears insulin dysregulation and abnormal central nervous system (CNS) insulin metabolism ${ }^{20}$ are also important independent factors. Insulin can cross the blood-brain barrier and is also produced in the CNS. Some studies have suggested a role for CNS insulin in controlling tau 
phosphorylation and protecting against $A \beta$ accumulation via insulin degrading enzyme, ${ }^{21}$ while others have demonstrated lower levels of insulin in the cerebrospinal fluid (CSF) of people with $\mathrm{AD}{ }^{22}$ The potential pathway for this involves peripheral hyperinsulinaemia downregulating insulin uptake across the blood-brain barrier because of oversaturation above physiological levels. ${ }^{23}$ This results in the downregulation of insulin-degrading enzyme in the brain, which mediates amyloid clearance. Less insulin signalling may also enhance the activity of the enzyme glycogen synthase kinase 3 , which can promote formation of tau and NFTs. ${ }^{24}$

These findings have led to the theory that drugs used in diabetes may be able to modify the pathophysiology of $\mathrm{AD}^{25}$ and a study of intranasal insulin as a therapy in mild cognitive impairment (MCI) and AD is currently being conducted after encouraging results from small pilot studies. ${ }^{26}$

\section{Apolipoprotein gene}

The Apo epsilon 4 (Apoe4) allele of the apolipoprotein (apo) gene, coding for a protein involved in cholesterol metabolism and lipid transport, has been identified as the primary genetic risk factor for $\mathrm{AD} .^{27}$ Individuals with one copy of the e 4 allele have a three-fold higher chance of developing $\mathrm{AD}$, while those with two alleles have an odds ratio of 14.9 for developing AD. ${ }^{28}$ The mechanism by which Apoe 4 increases the risk of AD remains unclear but it may act via its effect on $A \beta$ aggregation and clearance, thereby influencing the onset of $A \beta$ deposition. Other proposed mechanisms include effects on synaptic function, neurotoxicity, hyperphosphorylation of tau, and neuroinflammation. ${ }^{29}$ It has been proposed that modulation of the Apo-related receptor at the blood-brain barrier may offer a therapeutic target to affect $A \beta$ clearance, while other therapeutic options may include modulation of Apoe4 levels or converting Apoe4 to Apoe3, but clinical trials are currently lacking in this regard. ${ }^{30}$

\section{Neuroinflammation}

Neuroinflammation, an inflammatory response in the CNS characterised by accumulation of glial cells, appears to be a central event in AD pathophysiology. ${ }^{31}$ The brain was traditionally considered an 'immune-privileged' organ, isolated from the immune system by factors such as the blood-brain barrier and apparent inability of brain immune cells to mount an innate immune response, ${ }^{32}$ but opinion on this has subsequently shifted dramatically. In the 1990s, seminal epidemiological evidence suggested that antiinflammatory drugs may have a protective effect in $\mathrm{AD}^{33}$ and neuroinflammatory cascades are now considered an important target for treatment in $\mathrm{AD},{ }^{34}$ and both amyloid plaques and NFTs may act as drivers for this immune response.

Unfortunately, meta-analyses have demonstrated no benefit of non-steroidal anti-inflammatory drugs, aspirin or steroids over placebo in patients with already symptomatic AD; ${ }^{35}$ however, some evidence suggests that naproxen may have a role in prevention of $\mathrm{AD}$ in healthy older people. ${ }^{36}$ It may be that the therapeutic window for such treatment occurs early in the disease process and as such, by the time symptoms emerge, this opportunity has been lost. ${ }^{37}$

\section{Drug therapy}

Much of the research in AD in the last decade has been directed towards disease-modifying therapy that will alter the course of the disease rather than act on symptoms alone, however the lack of effective disease-modifying drugs arising from these studies reflects the challenges involved in developing a therapeutic agent with potential to modify the course of a disease as complex as AD. ${ }^{38}$

\section{Approved drug treatments}

\section{Cholinesterase inhibitors}

Tacrine was the first-generation cholinesterase inhibitor but was limited by hepatotoxic side effects. ${ }^{39}$ Donepezil, rivastigmine and galantamine then followed, with the former probably the most widely used agent.

Efficacy appears similar between these different agents so choice should be based on cost, individual patient tolerance and physician experience.

Donepezil is prescribed at an initial dose of $5 \mathrm{mg}$ in the evening, increased to $10 \mathrm{mg}$ after one month if appropriate. ${ }^{40}$ Response is gauged by a rating of better memory, function or behaviour by the patient or carer: there is no point in trying to measure change with brief mental status schedules such as the mini mental state examination, as these are not designed to detect clinically relevant change. If there is no response after three months of treatment it is reasonable to consider stopping the medicine at that stage although opinions around this can differ. Common side effects are gastrointestinal, fatigue and muscle cramps, and all patients should have an electrocardiogram prior to commencing a cholinesterase inhibitor because of the risk of sick sinus syndrome and other conduction abnormalities. Care should be taken if considering commencing a cholinesterase inhibitor in a person with a history of peptic or duodenal ulcer disease. Small numbers of patients may exhibit an acute worsening of cognition or agitation on starting; in which case, the medicine should be stopped immediately.

Average effects on cognition and function are generally modest ${ }^{41}$ and response rates are variable, with around onethird of patients showing no benefit and a smaller proportion (around one-fifth) showing larger benefit. It is expected that about one-third of patients may not tolerate a cholinesterase inhibitor because of side effects.

\section{Memantine}

Memantine uncompetitively blocks the NMDA receptor ${ }^{42}$ and, thus, may be neuroprotective by preventing neuron loss, as well as improving symptoms by helping to restore function of damaged neurons (Fig 1).

Memantine is initially prescribed at a dose of $5 \mathrm{mg}$ daily, increasing weekly by $5 \mathrm{mg}$ to a maximum dose of $20 \mathrm{mg} .{ }^{40}$ It is generally well tolerated, with fewer side effects than cholinesterase inhibitors, although dizziness, headache, somnolence, constipation and hypertension can occur.

Memantine has been shown to have modest benefits in moderate to severe $\mathrm{AD}$, with little evidence supporting its use in milder AD. ${ }^{43}$ Additionally, the addition of memantine to donepezil monotherapy may be beneficial in those with 
mid-stage AD or who are deteriorating cognitively. ${ }^{44}$ Neither memantine nor donepezil are beneficial in MCI.

While these medications represent our best current available pharmacological treatments in $\mathrm{AD}$, they have relatively small average overall effect and do not alter the course of the underlying neurodegenerative process. It is likely that the down regulation of cholinergic transmission occurs too far downstream in this process for treatments such as cholinesterase inhibitors to exhibit such an effect. ${ }^{45}$ With this in mind, targeting the pathological process 'upstream' has also been the focus of much attention (Fig 1).

\section{Potential future drug treatments}

\section{Anti-amyloid therapy}

Until recently, several high-profile clinical trials of pharmacological agents targeted at modifying this amyloid cascade have been undertaken, with largely disappointing results. These agents generally had three different target sites: directly targeting $A \beta$, and either the gamma or beta-secretase enzymes involved in APP cleavage. ${ }^{46}$

\section{Beta-secretase enzyme}

Small molecule beta-secretase inhibitors have demonstrated reduced CSF beta-amyloid compared to controls. Phase II/ III clinical trials of two agents, AZD3293 and MK-8931, are underway and due to be completed in $2019 .{ }^{47}$

\section{Gamma-secretase enzyme}

Phase III clinical trials of semagacestat, a small molecule gamma-secretase inhibitor, ${ }^{48}$ including over 3,000 patients, were discontinued in 2010 because of no improvement in cognition in the study group and worsening cognition at higher doses compared to controls. ${ }^{49}$ Incidence of skin cancer was also higher in the study group.

Tarenflurbil, related to the NSAID flurbiprofen, has been shown to reduce levels of $A \beta$ by modulating the gammasecretase enzyme, but demonstrated no improvement in cognition or function compared with placebo in phase III trials involving almost 1,700 patients. ${ }^{50}$

\section{Immunisation}

The initial human clinical trial of active immunisation against $\mathrm{A} \beta$ with the agent AN 1792 was stopped because of cases of meningoencephalitis in $6 \%$ of subjects. ${ }^{51}$ Subsequently, phase II trials of passive immunisation with intravenous immunoglobulin containing $A \beta$ antibodies in a small number of patients with mild to moderate $\mathrm{AD}$ has been shown to be safe and potentially efficacious but phase III studies found no evidence for slowing progression of $\mathrm{AD} .^{52}$

\section{Monoclonal antibodies}

Bapineuzumab, a monoclonal antibody to $A \beta,{ }^{53}$ underwent a phase III clinical trial from 2007 to 2012 in patients with mild to moderate $\mathrm{AD}$. It was shown to reduce the rate of amyloid accumulation in Apoe 4 carriers but did not demonstrate any treatment effect on either cognitive or functional outcomes despite engaging its target. ${ }^{54}$
However, another monoclonal antibody, solanezumab, completed two phase III trials in 2012 and failed to reach predefined end points in patients with mild to moderate AD. ${ }^{55}$ Subsequently, pooled analysis showed that cognitive scores in a subgroup of patients with milder symptoms showed small benefits and an extension study, where those previously taking placebo crossed over to solanezumab, was undertaken. The difference in cognitive scores between these two groups was sustained for a further two years, suggesting that, while the absolute benefit was small, solanezumab had a potentially disease-modifying effect during the placebo-controlled phase. $^{56}$

While this may represent the first evidence of disease modification on $\mathrm{AD}$, results must be interpreted with caution ${ }^{57}$ and EXPEDITION 3, a placebo-controlled trial in mild AD, is ongoing with the aim of clarifying the results seen in this cohort.

Another monoclonal antibody, aducanumab, has shown promising results in patients with pre-clinical and mild AD. Phase Ib studies of 165 patients with pre-clinical/mild AD, demonstrated dose-dependent reductions in brain $\mathrm{A} \beta$, as well as dose-dependent slowing of mini mental state examination and clinical dementia rating at one year and phase III studies are commencing soon. ${ }^{58}$

\section{Tau-targeted therapy}

Tau-targeted strategies that are currently in clinical trials include agents to prevent hyperphosphorylation, as well as those targeting microtubule stability and aggregation. ${ }^{59}$ Both lithium and valproic acid may act to inhibit tau phosphorylation ${ }^{60}$ but randomised controlled trials of these agents were negative. ${ }^{61}$ More recently, a phase II clinical trial of methylthioninium, a tau aggregation inhibitor, has demonstrated minor benefits in cognition in patients with both mild and moderate $\mathrm{AD}$ after 50 weeks therapy and there are plans to proceed to phase III trials. $^{62}$

\section{Future directions}

Studies of potentially disease-modifying therapy up to now have generally been undertaken in patients with clinically detectable, established disease, while mounting evidence suggests that the pathological changes associated with dementia begin to occur several years before the emergence of the clinical syndrome. ${ }^{63}$ It is possible then that pharmacological therapy may be more beneficial in this pre-clinical stage before the neurodegenerative process has been established. Techniques to provide earlier diagnosis are key to testing this theory in clinical trials, facilitating trials in presymptomatic phases.

\section{Early diagnosis}

Currently, earlier diagnosis of AD is primarily based on CSF and neuroimaging biomarkers, reflected in new research diagnostic criteria for AD.

\section{Cerebrospinal fluid biomarkers}

Reflecting the underlying neuropathology of AD, CSF markers of amyloid and tau are reliable diagnostic tools to detect dementia. CSF A 342 is decreased in patients with $\mathrm{AD}$, possibly 
because of deposition of the peptide in plaques. ${ }^{64}$ However, a decreased ratio of $A \beta 42 / A \beta 40$ appears to be a more reliable marker than $A \beta 42$ alone. Elevated total tau is a very sensitive marker for detection of $\mathrm{AD}$ but is also increased in other dementias, including $\mathrm{VaD}$ and frontotemporal dementia, while elevated phosphorylated tau, the major component of NFTs, is more specific than total tau. Combinations of these CSF markers have been used to improve diagnostic potential in early stages of $\mathrm{AD}$, for example, MCI patients with both low A $\beta 42$ and high tau levels were shown to have a substantially increased risk of developing AD ${ }^{65}$ Despite this, the discriminatory power of CSF biomarkers in the differential diagnosis remains somewhat suboptimal as a lone diagnostic test $^{66}$ and current strategies for presymptomatic evaluation involve combining results with neuroimaging findings.

\section{Neuroimaging}

Traditionally, structural neuroimaging in AD was used to rule out alternative diagnoses when presentations were atypical, eg brain tumours. However, functional imaging modalities such as $18 \mathrm{~F}$-fluorodeoxyglucose positron emission tomography (FDG-PET), are now able to detect loss of neuronal function in asymptomatic individuals by measuring cerebral metabolic rates of glucose metabolism (CMRglc), a surrogate marker for neuronal activity. ${ }^{67}$ Patients with early AD demonstrate reduced CMRglc in parietotemporal, frontal and posterior cingulate cortices. ${ }^{68}$ These changes have also been shown to precede the onset of symptoms in individuals genetically at risk for $\mathrm{AD},{ }^{69}$ as well as in patients with MCI. ${ }^{70}$ However, there is some overlap of the hypometabolic regions found in $\mathrm{AD}$ with those found in other dementia subtypes, and the additional use of amyloid PET, which can estimate amyloid plaque surface area, improves diagnostic accuracy. ${ }^{71}$

\section{Re-evaluating research diagnostic criteria}

The need for presymptomatic diagnosis of AD has dictated that new research diagnostic criteria have been proposed, formalising the view that $\mathrm{AD}$ exists on a continuum from the presymptomatic phase, to a symptomatic, pre-dementia phase (MCI) and then to $\mathrm{AD}^{72}$ In these new criteria, biomarkers are used to establish the presence of presymptomatic $\mathrm{AD}$ in research subjects with no or very subtle overt symptoms. Three pre-clinical stages of AD are proposed for research purposes: asymptomatic amyloidosis, asymptomatic amyloidosis with neurodegeneration, amyloidosis with neurodegeneration and subtle cognitive decline. ${ }^{73}$

These diagnostic criteria will facilitate a more formalised approach to the diagnosis of presymptomatic $\mathrm{AD}$ and provide a framework for studying early interventions in AD. While this definition of presymptomatic AD is certainly useful to, and will be primarily used in the research environment, we must also be mindful of the ethical implications of presymptomatic diagnosis of a disease with relatively ineffective available medical treatment.

\section{Better trial design}

Better selection of patients for clinical trials may yield more favourable clinical outcomes. For example, the 'mild to moderate' AD group may be too heterogeneous, and treatment effects within subgroups could be lost, as seen in the solanezumab trials. As well as earlier diagnosis, advanced biomarker analysis may also facilitate better selection of study subjects by allowing selection of patients with more uniform underlying pathology for targeted trials. This individualised approach would mirror several cancer therapies where bespoke treatment is targeted at specific patients, using markers such as HER2.

\section{Conclusion}

Given the rising prevalence of dementia, and the relative inadequacy of current available pharmacological treatment, the need to develop and implement new therapies is pressing. Recent results from trials of agents in AD with potential disease-modifying effects are encouraging but must also be interpreted with caution. Such medicines could potentially delay the onset of dementia and would therefore markedly reduce its prevalence and impact; ${ }^{74}$ however, currently we remain a good distance away from clinically available disease-modifying therapy. One would hope, however, that with advancing neuroimaging techniques and biochemical biomarkers, and an enhanced understanding of the underlying pathological processes involved, that this becomes a realistic goal in the near future.

In addition, while focus on the development of new therapies is very welcome, we must also be mindful that dementia is a multifaceted, complex disease, which by its nature directs a need for a multidisciplinary approach to care. Our focus in managing patients with dementia must remain well rounded and holistic, concentrating not just on pharmacological therapy but also on the complex biopsychosocial aspects of caring for this group of patients.

\section{References}

1 Brayne C, Davis D. Making Alzheimer's and dementia research fit for populations. Lancet 2012;380:1441-3.

2 Ngandu T, Lehtisalo J, Solomon A et al. A 2 year multidomain intervention of diet, exercise, cognitive training, and vascular risk monitoring versus control to prevent cognitive decline in at-risk elderly people (FINGER): a randomised controlled trial. Lancet 2015;385:2255-63.

3 Cummings JL, Morstorf T, Zhong K. Alzheimer's disease drugdevelopment pipeline: few candidates, frequent failures. Alzheimers Res Ther 2014;6:37.

4 Hardy J, Selkoe DJ. The amyloid hypothesis of Alzheimer's disease: progress and problems on the road to therapeutics. Science 2002;297:353-6.

5 Murphy MP, LeVine H. Alzheimer's disease and the amyloid-beta peptide. J Alzheimers Dis 2010;19:311-23.

6 Serrano-Pozo A, Frosch MP, Masliah E, Hyman BT. Neuropathological alterations in Alzheimer disease. Cold Spring Harb Perspect Med 2011;1:a006189.

7 Mandelkow EM, Mandelkow E. Tau in Alzheimer's disease. Trends Cell Biol 1998;8:425-7.

8 Bloom GS. Amyloid- $\beta$ and tau: the trigger and bullet in Alzheimer disease pathogenesis. JAMA Neurol 2014;71:505-8.

9 Huang HCC, Jiang ZFF. Accumulated amyloid-beta peptide and hyperphosphorylated tau protein: relationship and links in Alzheimer's disease. J Alzheimers Dis 2009;16:15-27.

10 Whitehouse PJ. The cholinergic deficit in Alzheimer's disease. J Clin Psychiatry 1998;59 Suppl 13:19-22. 
11 Lipton SA. The molecular basis of memantine action in Alzheimer's disease and other neurologic disorders: low-affinity, uncompetitive antagonism. Curr Alzheimer Res 2005;2:155-65.

12 Ravona-Springer R, Davidson M, Noy S. Is the distinction between Alzheimer's disease and vascular dementia possible and relevant? Dialogues Clin Neurosci 2003;5:7-15.

13 Hasnain M, Vieweg WV. Possible role of vascular risk factors in Alzheimer's disease and vascular dementia. Curr Pharm Des 2014;20:6007-13.

14 Kalaria RN, Ballard C. Overlap between pathology of Alzheimer disease and vascular dementia. Alzheimer Dis Assoc Disord 1999;13 Suppl 3:S115-23.

15 Petrovitch H, White LR, Izmirilian G et al. Midlife blood pressure and neuritic plaques, neurofibrillary tangles, and brain weight at death: the HAAS. Honolulu-Asia aging Study. Neurobiol Aging 2000;21:57-62.

16 Tolppanen AMM, Solomon A, Soininen H, Kivipelto M. Midlife vascular risk factors and Alzheimer's disease: evidence from epidemiological studies. J Alzheimers Dis 2012;32:531-40.

17 O’Brien JT, Markus HS. Vascular risk factors and Alzheimer's disease. BMC Med 2014;12.

18 Valenti R, Pantoni L, Markus HS. Treatment of vascular risk factors in patients with a diagnosis of Alzheimer's disease: a systematic review. BMC Med 2014;12:160.

19 Lu FPP, Lin KPP, Kuo HKK. Diabetes and the risk of multi-system aging phenotypes: a systematic review and meta-analysis. PLoS One 2009; $4: \mathrm{e} 4144$

20 Luchsinger JA, Tang MXX, Shea S, Mayeux R. Hyperinsulinemia and risk of Alzheimer disease. Neurology 2004;63:1187-92.

21 Farris W, Mansourian S, Chang Y et al. Insulin-degrading enzyme regulates the levels of insulin, amyloid beta-protein, and the betaamyloid precursor protein intracellular domain in vivo. Proc Natl Acad Sci USA 2003;100:4162-7.

22 Craft S, Peskind E, Schwartz MW et al. Cerebrospinal fluid and plasma insulin levels in Alzheimer's disease: relationship to severity of dementia and apolipoprotein E genotype. Neurology 1998;50:164-8.

23 Banks WA, Jaspan JB, Huang W, Kastin AJ. Transport of insulin across the blood-brain barrier: saturability at euglycemic doses of insulin. Peptides 1997;18:1423-9.

24 Martinez A, Gil C, Perez DI. Glycogen synthase kinase 3 inhibitors in the next horizon for Alzheimer's disease treatment. Int $J$ Alzheimers Dis 2011;2011:280502.

25 Yarchoan M, Arnold SE. Repurposing diabetes drugs for brain insulin resistance in Alzheimer disease. Diabetes 2014;63:2253-61.

26 Craft S, Baker LD, Montine TJ et al. Intranasal insulin therapy for Alzheimer disease and amnestic mild cognitive impairment: a pilot clinical trial. Arch Neurol 2012;69:29-38.

27 Corder EH, Saunders AM, Strittmatter WJ et al. Gene dose of apolipoprotein E type 4 allele and the risk of Alzheimer's disease in late onset families. Science 1993;261:921-3.

28 Farrer LA, Cupples LA, Haines JL et al. Effects of age, sex, and ethnicity on the association between apolipoprotein E genotype and Alzheimer disease. A meta-analysis. APOE and Alzheimer Disease Meta Analysis Consortium. JAMA 1997;278:1349-56.

29 Kim J, Basak JM, Holtzman DM. The role of apolipoprotein E in Alzheimer's disease. Neuron 2009;63:287-303.

30 Liu CCC, Liu CCC, Kanekiyo T, Xu H, Bu G. Apolipoprotein E and Alzheimer disease: risk, mechanisms and therapy. Nat Rev Neurol 2013;9:106-18.

31 Morales I, Guzmán-Martínez L, Cerda-Troncoso C, Farías GA, Maccioni RB. Neuroinflammation in the pathogenesis of Alzheimer's disease. A rational framework for the search of novel therapeutic approaches. Front Cell Neurosci 2014;8:112.

32 Carson MJ, Doose JM, Melchior B, Schmid CD, Ploix CC. CNS immune privilege: hiding in plain sight. Immunol Rev 2006;213:4865.
33 McGeer PL, Schulzer M, McGeer EG. Arthritis and anti-inflammatory agents as possible protective factors for Alzheimer's disease: a review of 17 epidemiologic studies. Neurology 1996;47:425-32.

34 Heneka MT, Carson MJ, El Khoury J et al. Neuroinflammation in Alzheimer's disease. Lancet Neurol 2015;14:388-405.

35 Jaturapatporn D, Isaac MGEKNG, McCleery J, Tabet N. Aspirin, steroidal and non-steroidal anti-inflammatory drugs for the treatment of Alzheimer's disease. Cochrane Database Syst Rev 2012;2:CD006378.

36 Breitner JC, Baker LD, Montine TJ et al. Extended results of the Alzheimer's disease anti-inflammatory prevention trial. Alzheimers Dement 2011;7:402-11.

37 Hensley K. Neuroinflammation in Alzheimer's disease: mechanisms, pathologic consequences, and potential for therapeutic manipulation. Alzheimers Dement 2010;21:1-14.

38 Salomone S, Caraci F, Leggio GMM et al. New pharmacological strategies for treatment of Alzheimer's disease: focus on disease modifying drugs. Br J Clin Pharmacol 2012;73:504-17.

39 Manning FC. Tacrine therapy for the dementia of Alzheimer's disease. Am Fam Physician 1994;50:819-26.

40 National Institute for Health and Care Excellence. Donepezil, galantamine, rivastigmine and memantine for the treatment of Alzheimer's disease. London: NICE, 2011. Available online at www.nice.org.uk/ guidance/ta217/chapter/3-The-technologies [Accessed 22 December 2015].

41 Birks JS, Harvey R. Donepezil for dementia due to Alzheimer's disease. Cochrane Database Syst Rev 2003;(3):CD001190.

42 Robinson DM, Keating GM. Memantine: a review of its use in Alzheimer's disease. Drugs 2006;66(11):1515-34.

43 Areosa SA, Sherriff F. Memantine for dementia. Cochrane Database Syst Rev 2003; (3): CD003154.

44 Atri A, Molinuevo JL, Lemming $\mathrm{O}$ et al. Memantine in patients with Alzheimer's disease receiving donepezil: new analyses of efficacy and safety for combination therapy. Alzheimers Res Ther 2013;5:6.

45 Mufson EJ, Counts SE, Perez SE, Ginsberg SD. Cholinergic system during the progression of Alzheimer's disease: therapeutic implications. Expert Rev Neurother 2008;8:1703-18.

46 Ghezzi L, Scarpini E, Galimberti D. Disease-modifying drugs in Alzheimer's disease. Drug Des Dev Ther 2013;7:1471-8.

47 Vassar R. BACE1 inhibitor drugs in clinical trials for Alzheimer's disease. Alzheimers Res Ther 2014;6:89.

48 Henley DB, May PC, Dean RA, Siemers ER. Development of semagacestat (LY450139), a functional gamma-secretase inhibitor, for the treatment of Alzheimer's disease. Exp Opin Pharmacother 2009;10:1657-64.

49 Doody RS, Raman R, Farlow M et al. A phase 3 trial of semagacestat for treatment of Alzheimer's disease. $N$ Engl J Med 2013;369:341-50.

50 Green RC, Schneider LS, Amato DA et al. Effect of tarenflurbil on cognitive decline and activities of daily living in patients with mild Alzheimer disease: a randomized controlled trial. JAMA 2009;302:2557-64.

51 Robinson SR, Bishop GM, Lee HGG, Münch G. Lessons from the AN 1792 Alzheimer vaccine: lest we forget. Neurobiol Aging 2004;25:609-15.

52 Loeffler DA. Intravenous immunoglobulin and Alzheimer's disease: what now? J Neuroinflammation 2013;10:70.

53 Prins ND, Scheltens P. Treating Alzheimer's disease with monoclonal antibodies: current status and outlook for the future. Alzheimers Res Ther 2013;5;56.

54 Salloway S, Sperling R, Fox NC et al. Two phase 3 trials of bapineuzumab in mild-to-moderate Alzheimer's disease. $N$ Engl J Med 2014;370:322-33.

55 Doody RS, Thomas RG, Farlow M et al. Phase 3 trials of solanezumab for mild-to-moderate Alzheimer's disease. $N$ Engl J Med 2014;370:311-21. 
56 Siemers ER, Sundell KL, Carlson C et al. Phase 3 solanezumab trials: Secondary outcomes in mild Alzheimer's disease patients. Alzheimers Dement 2016:12:110-20.

57 McCartney M. Margaret McCartney: The "breakthrough" drug that's not been shown to help in Alzheimer's disease. BMJ 2015;351:h4064.

58 National Institute on Aging. US Department of Health and Human Services. BIIB037 in prodromal or mild Alzheimer's disease. Bethesda, MD: NIA, 2016. Available online at www.nia.nih.gov/alzheimers/ clinical-trials/biib037-prodromal-or-mild-alzheimers-disease [Accessed 5 April 2016]

59 Wischik CM, Harrington CR, Storey JM. Tau-aggregation inhibitor therapy for Alzheimer's disease. Biochem Pharmacol 2014;88:529-39.

60 Tariot PN, Aisen PS. Can lithium or valproate untie tangles in Alzheimer's disease? J Clin Psychiatry 2009;70:919-21.

61 Hampel H, Ewers M, Bürger K et al. Lithium trial in Alzheimer's disease: a randomized, single-blind, placebo-controlled, multicenter 10-week study. J Clin Psychiatry 2009;70:922-31.

62 Wischik CM, Staff RT, Wischik DJ et al. Tau aggregation inhibitor therapy: an exploratory phase 2 study in mild or moderate Alzheimer's disease. J Alzheimers Dis 2015;44:705-20.

63 Braak H, Braak E. Frequency of stages of Alzheimer-related lesions in different age categories. Neurobiol Aging 1997;18:351-7.

64 Anoop A, Singh PK, Jacob RS, Maji SK. CSF Biomarkers for Alzheimer's disease diagnosis. Int J Alzheimers Dis 2010;2010:606802.

65 Hertze J, Minthon L, Zetterberg $\mathrm{H}$ et al. Evaluation of CSF biomarkers as predictors of Alzheimer's disease: a clinical follow-up study of 4.7 years. J Alzheimers Dis 2010;21:1119-28.

66 Engelborghs S, Le Bastard N. The impact of cerebrospinal fluid biomarkers on the diagnosis of Alzheimer's disease. Mol Diagn Ther 2012;16:135-41.

67 Mosconi L, Berti V, Glodzik L et al. Pre-clinical detection of Alzheimer's disease using FDG-PET, with or without amyloid imaging. J Alzheimers Dis 2010;20:843-54.
68 Mosconi L, Berti V, Glodzik L et al. Pre-clinical detection of Alzheimer's disease using FDG-PET, with or without amyloid imaging. J Alzheimers Dis 2010;20:843-54.

69 Kennedy AM, Frackowiak RS, Newman SK et al. Deficits in cerebral glucose metabolism demonstrated by positron emission tomography in individuals at risk of familial Alzheimer's disease. Neurosci Lett 1995;186:17-20

70 Chételat G, Desgranges B, de la Sayette V et al. Mild cognitive impairment: Can FDG-PET predict who is to rapidly convert to Alzheimer's disease? Neurology 2003;60:1374-7.

71 Vandenberghe R, Adamczuk K, Van Laere K. The interest of amyloid PET imaging in the diagnosis of Alzheimer's disease. Curr Opin Neurol 2013;26:646-55.

72 Jack CR, Albert MS, Knopman DS et al. Introduction to the recommendations from the National Institute on Aging-Alzheimer's Association workgroups on diagnostic guidelines for Alzheimer's disease. Alzheimers Dement 2011;7:257-62.

73 Croisile B, Auriacombe S, Etcharry-Bouyx F, Vercelletto M. National Institute on Aging (US), Alzheimer Association. [The new 2011 recommendations of the National Institute on Aging and the Alzheimer's Association on diagnostic guidelines for Alzheimer's disease: Preclinal stages, mild cognitive impairment, and dementia]. Rev Neurol (Paris) 2012;168:471-82.

74 Sloane PD, Zimmerman S, Suchindran C et al. The public health impact of Alzheimer's disease, 2000-2050: potential implication of treatment advances. Annu Rev Public Health 2002;23:213-31.

Address for correspondence: Dr R Briggs, Centre for Aging, Neuroscience and the Humanities, Trinity Centre for Health Sciences, Tallaght Hospital, Dublin, Ireland.

Email: briggsr@tcd.ie
Assessing trainees in the workplace An e-learning module for secondary care doctors

Three hours of CPD-approved interactive learning covering:

$>$ feedback

> supervised learning events (SLES)

$>$ workplace-based assessments (WPBAs)

$>$ the role of the Annual Review of Competence Progression (ARCP).

For more information please visit: www.rcplondon.ac.uk/elearning

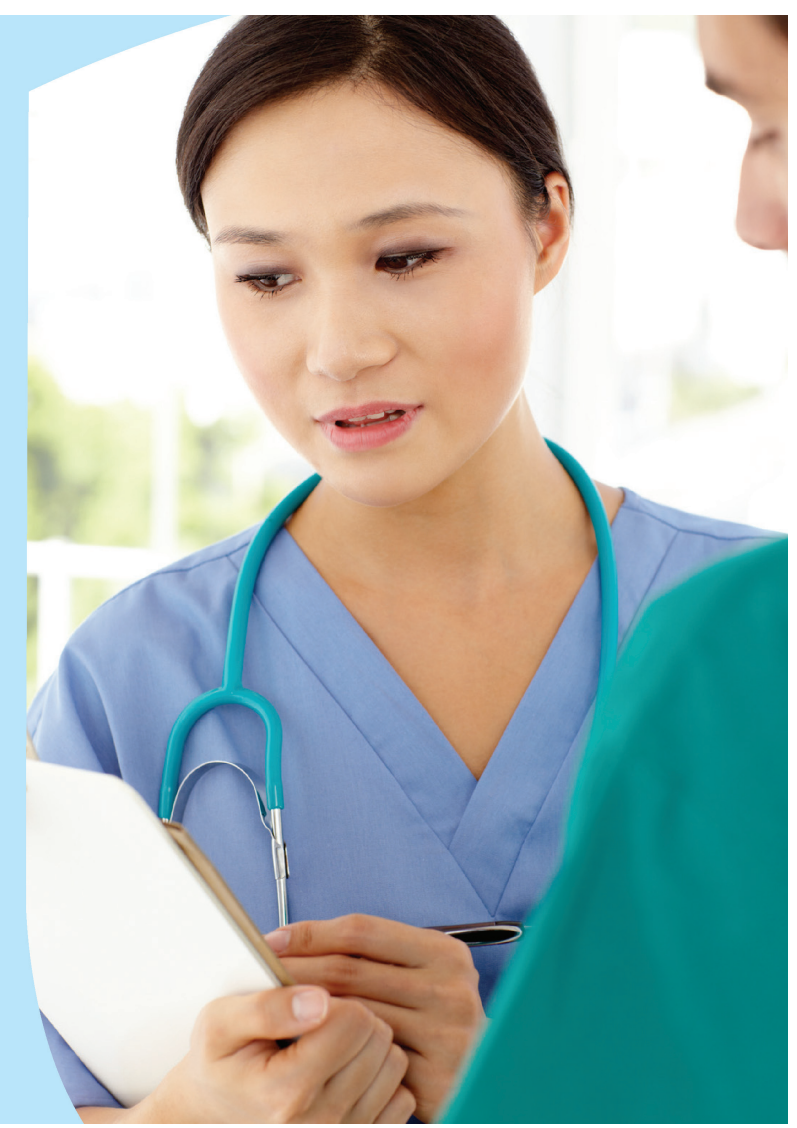

